\title{
Amotivación en la actividad física de la población mayor de 15 años de Monterrey(México)

\author{
Amotivation in physical activity of the population over 15 years of Monterrey (Mexico)
}

\author{
*Jorge Isabel Zamarripa Rivera, **Francisco Ruiz Juan, *Jeanette M. López Walle, *Marco Antonio Garrido Salazar \\ *Universidad Autónoma de Nuevo León, **Universidad de Murcia
}

Resumen: El objetivo de éste trabajo es describir las razones que han originado la amotivación para no haber hecho actividad física los regiomontanos adultos en función al sexo, la edad y el nivel de estudios. La muestra se estimó en 1,118 sujetos para obtener una validez en los resultados con un margen de error muestral del $\pm 3 \%$ y un nivel de confianza del 95,5\%. El procedimiento de muestreo utilizado ha sido polietápico con afijación proporcional, recurriendo a dos tipos de muestreo aleatorio estratificado y aleatorio por conglomerados. Los resultados revelan que las creencias de valor fueron las que generaron la amotivación para nunca haber realizado actividad física de tiempo libre de los sujetos de 30 a 44 años y con preparatoria.

Palabra clave: Inactividad física, Amotivación.

Abstract: The aim of this paper is to describe the reasons that have given origin to the amotivation of Monterrey adult citizens that have not done any physical activity according to gender, age and the level of studies. The sample was estimated in 1,118 subjects to obtain result validity with an error range of $\pm 3 \%$ and a reliability level of $95.5 \%$. The sample procedure used has been multistage with a proportional affixation, resorting to two types of stratificated random sampling by conglomerates. Results reveal that the values beliefs were the ones generating the amotivation to never have practiced any physical activity on leisure time of the subjects from 30 to 44 years old with high school finished.

Key words: Physical Inactivity, Amotivation.

\section{Introducción}

El estilo de vida sedentario permite la aparición de enfermedades cardiacas, algunos tipos de cáncer, diabetes tipo II, infarto de miocardio y ciertos desórdenes músculo esqueléticos (Booth, Chakravarthy, Gordon \& Spangenburg, 2002; García Pérez, García Roche, Pérez Jiménez \& Bonet Gorbea, 2007; Martinson, O’Connor \& Pronk, 2001). La prevalencia de estas enfermedades, son un grave problema para la salud pública, ya que una proporción considerable de la mortalidad, es ocasionada por las enfermedades crónicas no transmisibles más frecuentes.

En México, el porcentaje de mortalidad de las enfermedades crónicas no transmisibles supera el 40\% (Pan American Health Organization [PAHO], 2004). Asimismo, las estadísticas muestran que más del $40 \%$ de las muertes en Nuevo León, son ocasionadas por enfermedades que pueden ser prevenidas por medio de una práctica física regular, como las cardiovasculares, la diabetes mellitus y las cerebro-vasculares (Instituto Estatal de las Mujeres, Nuevo León [IEM], 2005).

Esta situación, supone un grave inconveniente para los gobernantes e instituciones responsables de la salud pública si tenemos en cuenta que uno de sus principales objetivos es el de mantener y optimizar la calidad de vida de la población, lo cual no es tarea fácil. Pero para conseguir esto es necesario mejorar la salud de sus habitantes (Vuori, Oja, Cavill \& Coumans, 2001).

Diferentes estudios (Alemán-Mateo, Esparza-Romero \& Valencia, 1999; Barrios Duarte, Borges Mojaiber \& Cardoso Pérez, 2003; Lobelo, Pate, Parra, Duperly \& Pratt, 2006; Marques, 1995; Vuori et al., 2001) e informes de salud (National Health Service Information Centre, Lifestyle Statistics [NHSICLS], 2009; United States Department of Health and Human Services [USDHHS], 1996; World Health Organization [WHO], 2008) han demostrado que las personas que realizan actividad física mejoran aspectos relacionados con beneficios a nivel físico, mental y social, los cuales se ven reflejados en su calidad de vida.

La mayoría de las personas aceptan esta relación entre la actividad física, la condición física saludable y una positiva calidad de vida, sin

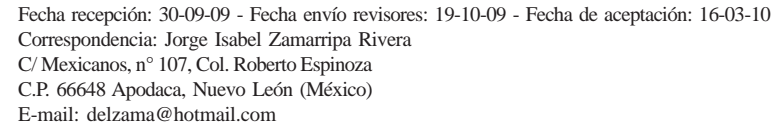

embargo y a pesar de que son conscientes de los bienes que genera el estilo de vida activo, muy pocos se comprometen con su participación.

Las razones que originan la práctica y aquellas que impiden la misma son diversas, Sallis y Owen (1999) después de una revisión literaria realizaron una clasificación de los correlatos asociados al ejercicio físico. En esta clasificación, identificaron al sexo, la edad y el nivel de estudios como fuertes indicadores de la actividad física.

Muchos estudios e informes de salud, han coincidido sobre la diferencia marcada por el sexo en su comportamiento ante la actividad física, donde la prevalencia de inactividad física es mayor en las mujeres que en los varones (Barnes \& Schoenborn, 2003; Burton \& Turrell, 2000; Castro-Carvajal, Patiño-Villada, Cardona-Rendón \& OchoaPatiño, 2008; CDCP, 2003, 2007; Curi, Gomes, Kingdon \& Costa, 2003; Florindo et al., 2009; Martínez-González et al., 2001; NHSICLS, 2009; Porras-Sanchez, 2009; Ruiz-Juan, de la Cruz \& Piéron, 2009; Ruiz-Juan \& García-Montes, 2005; Varo et al., 2003; WHO, 2003a, 2003b).

Igualmente, la edad es un factor fuertemente asociado con la inactividad física, los estudios realizados al respecto, mencionan que las personas mayores son menos activos que las personas más jóvenes (Borodulin, Laatikainen, Juolevi \& Jousilahti, 2008; Burton \& Turrell, 2000; Campbell, MacAuley, McCrum \& Evans, 2001; Curi et al., 2003; Eyler et al., 2003; Florindo et al., 2009; García-Ferrando, 2001; Martínez-González et al., 2001; Porras-Sanchez, 2009; Ruiz-Juan, de la Cruz et al., 2009; Ruiz-Juan \& García-Montes, 2005; Varo et al., 2003).

El nivel educativo de las personas también es un factor importante en los comportamientos ante la actividad física. Los estudios concluyen que las personas que no tienen o tienen un nivel educativo bajo, presentan tasas de inactividad física mayores que aquellos que presentan un nivel académico mayor, es decir, el nivel académico está inversamente relacionado con la práctica de actividad físico-deportiva(Castro-Carvajal et al., 2008; Florindo et al., 2009; Gómez, Duperly, Lucumí, Gámez \& Venegas, 2005; Haakstad \& Bø, 2007; Hui \& Morrow, 2001; MartínezGonzález et al., 2001; Seclén-Palacín \& Jacoby, 2003; Varo et al., 2003; Wilcox, Castro, King, Housemann \& Brownson, 2000).

Al igual que los factores biológicos y demográficos, existen otros factores de tipo psicológico que pueden originar la inactividad física comola amotivación.

El impulso de las personas para llevar a cabo comportamientos específicos pueden ser muy diferentes de aquellos factores responsables 
de su falta de voluntad. Por lotanto, una mejor comprensión del fenómeno de la amotivación puede contribuir a clarificar el proceso complejo que involucra realizar cierto comportamiento (Pelletier, Dion, Tuson \& Green-Demers, 1999).

Los individuos amotivados son incapaces de prevenir las consecuencias de sus comportamientos, por lo tanto, no perciben la base de sus motivos, así que ellos dudan de sus acciones y probablemente desistan en el futuro (Pelletier et al., 1999).

En este estudio, nos apoyaremos en la clasificación de la amotivación adaptada al ejercicio en personas mayores (Vlachopoulos \& Gigoudi, 2008), la cual se compone de las creencias de capacidad, es decir, la amotivación surge cuando un sujeto cree que no posee lo necesario para realizar satisfactoriamente un comportamiento (Bandura, 1977, 1982; Pelletier et al., 1999; Skinner, Wellborn \& Connell, 1990).

La amotivación por creencias de resultados surge cuando un sujeto cree que un comportamiento específico no será eficaz para lograr la meta deseada (Bandura, 1977, 1982; Pelletier et al., 1999).

La amotivación porcreencias de esfuerzos surge cuando las personas creen, piensan o sienten que no podrán ejercer el esfuerzo sostenible para realizar y mantener el comportamiento (Legault, Green-Demers \& Pelletier, 2006; Pelletier et al., 1999).

La amotivación por creencias de valor surge cuando la actividad o comportamiento a realizar no representa un componente integral en la vida de una persona (Legault et al., 2006).

El objetivo principal de este estudio es describir las razones que han originado la amotivación para nunca haber sido activo en el tiempo libre de los regiomontanos adultos en función al sexo, la edad y el nivel de estudios.

\section{Material y método}

\subsection{Población y muestra}

En el presente estudio, participaron una muestra de 1,118 sujetos mayores de 14 años de Monterrey, Nuevo León, México. Los datos de esta investigación han sido obtenidos a través de la información proporcionada por el INEGI, en febrero de 2007, atendiendo al XII Censo General de Población y Vivienda 2000 (Instituto Nacional de Estadística, Geografía e Informática [INEGI], 2005).

Éstos estaban distribuidos atendiendo al número de habitantes por distritos censales (área geo-estadística básica[AGEB], según codificación numérica). Igualmente, se nos proporcionó la distribución de la población por sexo y edad (15-29, 30-44, 45-59 y 60 años o más). A partir de estos datos, extrajimos el total de la población censada en Monterrey que asciende a 808,632 habitantes de los cuales 393,923 son varones y 414,709 mujeres.

Toda investigación que contemple una población tan numerosa hace imposible que se pueda llegar a sondear a todos los individuos que la componen. Por ello, las técnicas estadísticas nos permiten acotar este número a otro mucho inferior (muestra) permitiéndonos, posteriormente, extrapolar la información recogida a toda la población. Es preciso, por lo tanto, recurrir a este tipo de procedimiento en el que la muestra, siendo lo suficientemente amplia, permita validar los resultados obtenidos con un error muestral pequeño y con un nivel de confianza alto.

Teniendo en cuenta esto, en este estudio, el tamaño de la muestra se estimó en 1,118 sujetos para obtener una validez en los resultados con un margen de error muestral del $\pm 3 \%$ y un nivel de confianza del $95,5 \%$. El procedimiento de muestreo utilizado ha sido polietápico con afijación proporcional, recurriendo a dos tipos de muestreo: el muestreo aleatorio estratificado y el muestreo aleatorio por conglomerados.

\subsection{Instrumento}

Para obtener la información necesaria en esta investigación se utilizó el cuestionario denominado «Hábitos físico-deportivos y estilos de vida» (Ruiz-Juan, García-Montes \& Piéron, 2009). Los bloques temáticos del cuestionario son: disponibilidad y ocupación del tiempo libre, valoración de la educación física recibida, hábitos físico-deportivos de tiempo libre (interés, práctica y demanda de actividades físicodeportivas), percepción de la condición física y del estado de salud, enfermedades más habituales y hábitos de consumo (alimentación, medicamentos, alcohol, tabaco, otras drogas). Para el presente trabajo se han considerado las variables sobre por los que nunca se ha realizado actividad físico-deportiva, así como el sexo, la edad y el nivel de estudios.

El cuestionario, cuenta con validez de contenido y de constructo. Igualmente, reseñar que hemos constatado la fiabilidad del cuestionario, mediante los diferentes estudios piloto, así como por las diferentes aportaciones hechas por los expertos que han colaborado en la investigación.

Para conocer el porqué de su inactividad, los sujetos inactivos indicaron en una escala compuesta por 11 ítems, el grado de influencia acerca de las barreras relacionadas a nunca haber hecho práctica física de tiempo libre, es decir, las razones por las cuales las personas nunca han hecho actividad física. Las respuestas se recogieron en una escala de tipo Likert de 4 puntos que oscila desde 1 (nada) a 4 (mucho).

\section{Resultados}

3.1. Motivos por los que nunca se ha realizado actividad física 3.1.1. Propiedades psicométricas de la escala de motivos para nunca haber practicado actividad física en la población inactiva

Se llevó a cabo un análisis factorial exploratorio a la escala antes mencionada. El coeficiente KMO fue de .765, lo que en este caso, nos indica un grado de adecuación muestral mediano, ya que el grado de variabilidad total de la muestra está explicado en un 76,5\% El resultado obtenido mediante la prueba de Bartlett (Prueba de Bartlett $=650.35, \mathrm{gl}$ $=55, p<.000$ ) mostró que no existe relación lineal entre los factores de la escala.

El análisis de componentes principales se llevó a cabo por medio de una rotación Varimax Kaiser utilizando como criterio de saturación significativa en los factores 0,40 . Este análisis dio como resultado tres componentes con valor propio mayor que la unidad. El factor 1 obtuvo un eigenvalue de 3,170, lo que representa un 28,81\% de la varianza total. El factor 2 obtuvo un eigenvalue de 1,608, lo que representa un 14,61\% de la varianza total. El factor 3 obtuvo un eigenvalue de 1,037, lo que representa un 9,42\% de la varianza total. El porcentaje acumulado de los tres factores explica el 52,86\% de la varianza.

La rotación Varimax Kaiser dio como resultado, tres subconjuntos o factores claramente diferenciados de variables, reflejados en la Tabla 1.

\subsubsection{Fiabilidad}

El coeficiente del Alfa de Cronbach muestra una alta consistencia interna del conjunto de estas 11 variables ya que presenta un valor deá $=.78$. De acuerdo al análisis factorial exploratorio, se decidió calcular en cada uno de los cuatro factores resultantes el coeficiente alfa, para conocer el grado de consistencia interna que presentaba cada factor, lo que nos permite pasar a un conjunto de tres nuevas variables o factores, no directamente observables.

Tabla 1 Cargas factoriales por componentes

\begin{tabular}{|c|c|c|c|c|}
\hline \multirow[b]{2}{*}{ No. } & \multirow[b]{2}{*}{ Ítems } & \multicolumn{3}{|c|}{ Componente } \\
\hline & & 1 & 2 & 3 \\
\hline 10 & Sus padres no se lo inculcaron o no le dejaban & ,792 & & \\
\hline 9 & Mis amigos no hacen & 673 & & \\
\hline 14 & Nadie me apoyo para iniciarme & 611 & & \\
\hline 11 & No me gusta la actividad físico-deportiva & 609 & & \\
\hline 3 & $\begin{array}{l}\text { Sus experiencias y vivencias en clases de educación física no fueron muy } \\
\text { agradables }\end{array}$ & & ,741 & \\
\hline 4 & No se me da bien el deporte & & 683 & \\
\hline 2 & No le enseñaron en el centro de estudios & & ,590 & \\
\hline 1 & No le veo ut ilidad y/o beneficios & & ,579 & \\
\hline 6 & No tengo tiempo & & & 816 \\
\hline 8 & Salgo muy cansado del trabajo odel estudio & & & 764 \\
\hline 7 & No hay instalaciones cerca $y / o$ adecuadas & & & ,561 \\
\hline
\end{tabular}




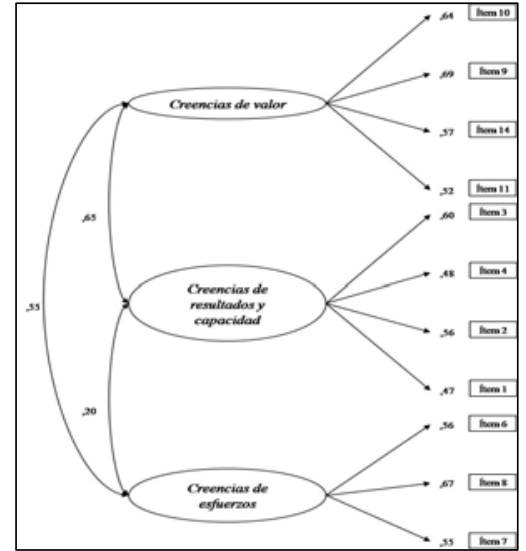

Figura. 1. Parámetros estandarizados de la escala.

El primer factor (componente 1) lo componen cuatro variables relativas a las barreras sociales y al valor negativo que da el sujeto a las actividades físicas provocando su inactividad, incluyendo los ítems: 10 , 9,14 y 11. Para éste primer factor, el cual denominaremos creencias de valor el puntaje obtenido fue de .70, muy cerca de los valores considerados como respetables.

En el segundo factor (componente 2) se concentran cuatro ítems relativos a la percepción sobre su capacidad y a los resultados esperados de la práctica física que pueden ocasionar la inactividad físico-deportiva en el tiempo libre de los sujetos, estos son: 3, 4, 2 y 1 . El Alfa de Conbrach para este factor denominado creencias de resultados $y$ capacidad, fue de 60 .

Finalmente, el tercer factor (componente 3) compuesto de tres ítems relacionados al esfuerzo que requiere la práctica física y que el sujeto no está dispuesto a asumir provocando la inactividad físicodeportiva, se refieren a los ítems: 6, 8 y 7. En este grupo, el cual llamaremos, creencias de esfuerzos obtuvo un valor de .62.

3.2. Análisis factorial confirmatorio de la escala de los motivos por los que nunca han realizado actividad física

Después de realizar el análisis factorial exploratorio, se procedió a realizar el análisis factorial confirmatorio. Debido a que el modelo no cumple con el supuesto de normalidad multivariante, se utilizó la técnica libre de distribución asintótica como método de estimación

La bondad del ajuste de la escala fue medida empleando los índices de ajuste absoluto son el Chi cuadrado $\left(\div^{2}\right), \div^{2} / g l$; el índice de bondad de ajuste (GFI); y la raíz del promedio del error de aproximación (RMSEA).

Los índices de bondad de ajuste para este modelo son adecuados, ya que diferencia encontrada entre el valor de discrepancia mínima de la muestra (CMIN) y el grado de libertad (DF) fue estadísticamente significativa y el $\div 2 /$ gl es menor a 5 . El valor presentado para el índice de bondad del ajuste (GFI) fue de 0,94, logrando la consideración de aceptable. Finalmente, el índice de la raíz cuadrada media del error de la aproximación(RMSEA) nos indica un error razonable de aproximación a la población.

El modelo evaluado presenta tres factores denominados: creencias de valor, creencias de resultados y capacidad, creencias de esfuerzos, las cuales permiten la validación del modelo teórico sobre las amotivaciones en sujetos físicamente inactivos de (Vlachopoulos \& Gigoudi, 2008) y que se representa en la siguiente Figura 1.

\subsection{Tipos de amotivaciones por las que nunca se ha realizado} actividad física

\subsubsection{Por sexo}

En nuestra población, las amotivaciones para nunca haber hecho actividad física no presentan una asociación con el sexo del sujeto en nuestra población. Es decir, el hecho de ser hombre o mujer, es un factor que no incide en la conducta físicamente inactiva, o para nunca haber realizado actividad física por creencias de valor, por creencias de resultados y capacidad o por creencias de esfuerzo.

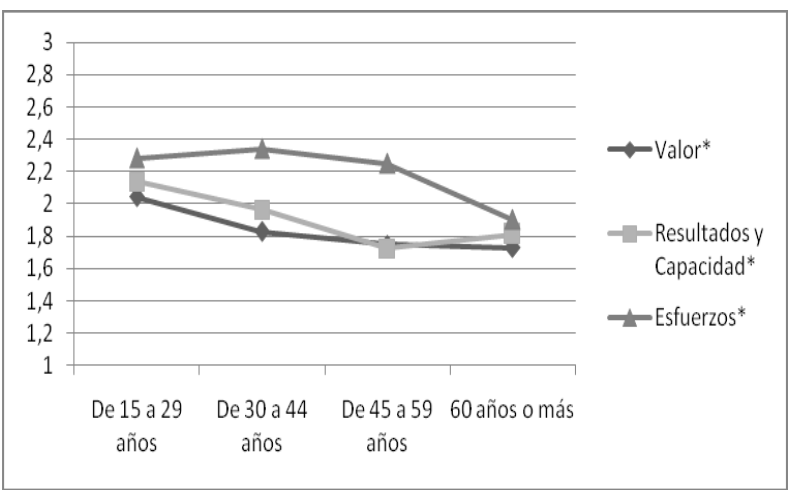

Figura. 2. Amotivaciones por las que nunca se ha realizado actividad física. Diferencias según los grupos de edad.

\subsubsection{Por edad}

La edad de los sujetos presentó diferencias significativas con la amotivación por las tres tipos de creencias para nunca haber realizado actividad física.

Respecto a las creencias de valor, observamos una diferencia notable entre los sujetos entre los 15 y 29 y aquellos que tienen 60 años o más, siendo los más jóvenes quienes mostraron altos niveles para nunca haber realizado actividad física por este tipo de creencias.

Por otro lado, la creencia de resultados y de capacidad mostró una diferencia considerable entre los sujetos con 15 y 29 años y aquellos entre los 44 y 59 años de edad, siendo de nuevo los más jóvenes quienes manifestaron en mayor medida que nunca habían realizado actividad física por este tipo de creencia.

Finalmente, las creencias de esfuerzos para nunca haber hecho actividad física, fueron más relevantes entre los sujetos de 30 a 44 años. Estas personas, creen que la práctica física no es tan importante como para realizar el esfuerzo necesario para ser físicamente activo (Figura 2).

\subsubsection{Por nivel de estudios}

Con relación al nivel de estudio, las creencias de valory las creencias de esfuerzos mostraron diferencias significativas.

La Figura 3, nos revela que las personas con estudios preparatorios terminados son las que, en mayor medida, nunca han hecho actividad física por estos tipos de creencias en comparación con aquellos que no tenían o tenían sólo la primaria. Por lo tanto, el poco valor atribuido a la práctica por estos sujetos y creer que no podrá sostener el esfuerzo necesario, puede estar relacionado al compromiso que actualmente requiere su formación universitaria.

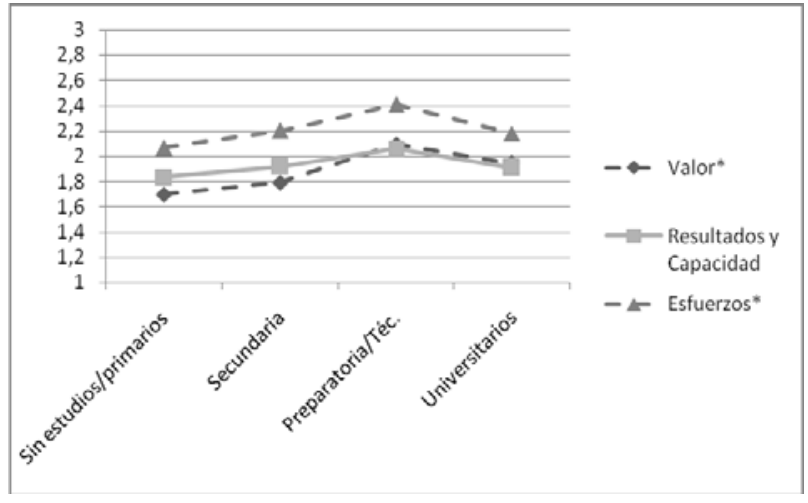

Figura.3. Amotivaciones por las que nunca se ha realizado actividad física. Diferencias según el nivel de estudios. 


\section{Discusión}

Nuestros resultados arrojaron datos interesantes sobre la amotivación para nunca haber realizado actividad físico-deportiva en el tiempo libre, ésta fue analizada sobre tres tipos de creencias: creencias de valor, creencias de resultados y capacidad y creencias de esfuerzos.

Nuestros resultados no mostraron diferencias significativas sobre los tipos de amotivaciones y el sexo de los sujetos. Esto nos indica que, los regiomontanos que nunca han realizado actividad física, no muestran una variación considerable entre los varones y las mujeres y creer que la práctica física no es lo suficientemente valiosa, creer que no le dará resultados esperados, creer que no es capaz de realizar eficientemente cierta actividad o creer que el esfuerzo que va desplegar es demasiado.

Sin embargo, otros estudios como el de Netz y Raviv (2004) y Daley y Duda (2006), las mujeres mostraron mayor desmotivación que los varones.

Con relación a los grupos de edad, nuestros resultados mostraron diferencias significativas en los tres tipos de creencias.

El grupo de 30 a 44 años, mostró mayor desmotivación por creencias de valor que el resto de los grupos. Está situación puede estar asociada con los compromisos que este grupo de personas suele tener, por ejemplo, la responsabilidad familiar y laboral, restándole valor a la práctica física.

Por otro lado, las creencias de resultados y capacidad, influyeron más en el grupo más joven que en el resto de los grupos de edad. Es decir, las creencias de resultados y capacidad, fueron las causas más citadas por el grupo regiomontano más joven que nunca ha realizado actividad física.

Posiblemente, esta situación se presenta en los jóvenes regiomontanos porque las experiencias y vivencias dentro de las clases de educación física no fueron agradables, lo que pudo provocar que el sujeto se crea incapaz de realizar alguna actividad físico-deportiva o que simplemente no le vea utilidad y beneficios.

Los resultados del estudio de Biddle, Wang, Chatzisarantis y Spray (2003) coinciden parcialmente con nuestros resultados al revelar que los sujetos con baja percepción de competencia presentan mayor amotivación que aquellos con una alta percepción de competencia para la actividad física y el deporte.

Por el contrario, y teniendo en cuenta que la auto-eficacia es considerada como los juicios de una persona sobre qué tan bien puede ejecutar los cursos de acción necesarios para afrontar una futura situación (Bandura, 1977), nuestros resultados sobre las creencias de capacidad contrastan con los de Netz y Raviv (2004), ya que los más viejos fueron los que percibieron menos auto-eficacia para la actividad física que los más jóvenes.

Finalmente, los sujetos del grupo de 30 a 44 años de edad que nunca ha realizado actividad física, estuvieron más desmotivados por creencias de esfuerzos que el resto de los grupos de edad. Es decir, la creencia de que la actividad física requiere de demasiado esfuerzo como para llevarla a cabo, es una opinión que los regiomontanos entre 30 y 44 años de edad refieren para nunca haber realizado actividad física.

Los estudios que han examinado las creencias de esfuerzos y la edad difieren con nuestros resultados, ya que los adultos mayores que nunca han sido activos manifiestan su desmotivación por creer que la actividad física requiere demasiado esfuerzo (Netz \& Raviv, 2004; Thøgersen-Ntoumani, Ntoumanis \& Nikitaras, 2008).

Es probable que en el momento de la entrevista, la mayor parte de los regiomontanos entre 30 y 44 años tuviera una ocupación laboral, ya que la mayoría de personas con esta edad, ya terminaron sus estudios o no se han jubilado. Estas personas, posiblemente terminen muy cansados de su trabajo, o perciben que las instalaciones para realizar actividad física adecuadamente se encuentran demasiado lejos como para dedicarle el tiempo y esfuerzo necesario para trasladarse y llevar a cabo su práctica física.

Por otro lado, las creencias de valor y de esfuerzos presentaron diferencias significativas entre los diferentes niveles de estudios. Nuestros resultados indican que, principalmente los sujetos con preparatoria terminada, nunca han realizado actividad física porque creen que no es lo suficientemente valiosa para su práctica regular y porque consideran que la actividad física no es lo suficientemente valiosa.

Cabe señalar que no se localizaron estudios que indagaran sobre estas variables, lo que nos ha impedido realizar las comparaciones pertinentes.

Sin embargo, posiblemente estas personas no recibieron, ni reciben apoyo de sus padres, amigos o cualquier otra persona para realizar actividad físico-deportiva, provocando su desmotivación para nunca haber realizado ni experimentado la práctica de actividad física, lo que ha impedido disfrutar y desarrollar el gusto por la práctica física, restándole valor a la misma.

\section{Bibliografía}

Alemán-Mateo, H., Esparza-Romero, J. \& Valencia, M. (1999). Antropometría y composición corporal en personas mayores de 60 años. Importancia de la actividad física. Salud Pública de México, 41, 309-316.

Bandura,A. (1977). Self-efficacy: Toward a unifying theory of behavioral change. Psychological Review, 84, 191-215.

Bandura,A.(1982). Self-efficacy mechanism in human agency.American Psychologist, 37, 122-147.

Barnes, P. \& Schoenborn, C. (2003). Physical Activity Among Adults: United States, 2000. Advance data from vital and health statics, 333.

Barrios-Duarte, R., Borges-Mojaiber, R., \& Cardoso-Pérez, L. (2003). Beneficios percibidos por adultos mayores incorporados al ejercicio. Revista Cubana de Medicina General Integral, 19.

Biddle, S., Wang, C., Chatzisarantis, N. \& Spray, C. (2003). Motivation for physical activity in young people: entity and incremental beliefs about athletic ability. Journal of Sports Sciences, 21, 973-989.

Booth, F., Chakravarthy, M., Gordon, S. \& Spangenburg, E. (2002). Waging war on physical inactivity: using modern molecular ammunition against an ancient enemy. Journal of Applied Physiology, 93, 3-30.

Borodulin, K., Laatikainen, T., Juolevi, A. \& Jousilahti, P. (2008). Thirtyyear trends of physical activity in relation to age, calendar time and birth cohort in Finnish adults. The European Journal of Public Health, 18, 339-344.

Burton, N. \& Turrell, G. (2000). Occupation, Hours Worked, and Leisure-Time Physical Activity. Preventive Medicine, 31, 673681.

Campbell, P., MacAuley, D., McCrum, E. \& Evans, A. (2001). Age differences in the motivating factors for exercise. / Differences d " age dans les facteurs motivationels pour l ' exercice. Journal of Sport \& Exercise Psychology, 23, 191-199.

Castro-Carvajal, J., Patiño-Villada, F., Cardona-Rendón, B., \& OchoaPatiño, V. (2008). Aspectos Asociados a la Actividad Física en el Tiempo Libre en la Población Adulta de un Municipio Antioqueño. (Spanish). Aspects associated with physical activity in the adult population's leisure time in a municipality in the Antioquia Department, Colombia. (English). Revista de Salud Pública, 10,679690.

Centers for Disease Control and Prevention (2003). Behavioural Risk Factor Surveillance System. Atlanta: National Center for Chronic Disease Prevention and Health.

Centers for Disease Control and Prevention (2007). National Average: Recommended Physical Activity by: Gender. Atlanta.

Curi, P., Gomes, C., Kingdon, J. \& Costa, R. (2003). Physical Inactivity: Prevalence and Associated Variables in Brazilian Adults. Medicine \& Science in Sports \& Exercise, 35, 1894-1900.

Daley, A., \& Duda, J. (2006). Self-determination, stage of readiness to change for exercise, and frequency of physical activity in young people. European Journal of Sport Science, 6, 231-243. 
Eyler, A., Matson-Koffan, D., Rohm, D., Wilcox, S., Wilbur, J., Thompson, J., et al. (2003). Cuantitative Study of Correlates of Physical Activity in Women from Diverse Racial/Ethnic Groups The Women's Cardiovascular Health Network Project Summary and Conclusions. American Journal of Preventive Medicine., 25(3Si), 93-103.

Florindo, A., Guimarães, V., Cesar, C., de Azevedo Barros, M., Alves, M. \& Goldbaum, M. (2009). Epidemiology of Leisure, Transportation, Occupational, and Household Physical Activity: Prevalence and Associated Factors. Journal of Physical Activity and Health, 6, 625-632.

García-Pérez, R., García-Roche, R., Pérez-Jiménez, D. \& Bonet-Gorbea, M.(2007). Sedentarismo y su relación con la calidad de vida relativa a salud: Cuba, 2001. Revista Cubana de Higiene y Epidemiología, 45.

García-Ferrando, M. (2001). Los españoles y el deporte: Prácticas y comportamientos en la última década del siglo XX (Encuesta de los hábitos deportivos de los españoles, 2000). Madrid: Ministerio de educación, cultura y deporte. Consejo superior de deportes.

Gómez, L., Duperly, J., Lucumí, D., Gámez, R. \& Venegas, A. (2005). Nivel de actividad física global en la población adulta de Bogotá (Colombia): Prevalencia y factores asociados. Gaceta Sanitaria, 19, 206-213.

Haakstad, L. \& Bø, K. (2007). Fitness and physical activity in Norwegian adults. Advances in Physiotherapy, 9(2), 89-96.

Hui, C. \& Morrow, J. (2001). Level of participation and knowledge of physical activity in Hong Kong Chinese adults and their association with age. / Niveau de participation et de l ' activite physique de chinois adultes de Hong Kong et leur rapport avec l ' age. Journal of Aging \& Physical Activity, 9, 372-385.

Instituto Nacional de Estadística, Geografía e Informática. (2005). Población total por AGEB según el sexo y los grupos seleccionados de edad. [CD-ROM]. México: Instituto Nacional de Estadística, Geografía e Informática.

Legault, L., Green-Demers, I. \& Pelletier, L. (2006). Why Do High School Students Lack Motivation in the Classroom? Toward an Understanding of Academic Amotivation and the Role of Social Support. Journal of Educational Psychology, 98, 567-582.

Lobelo, F., Pate, R., Parra, D., Duperly, J. \& Pratt, M. (2006). Carga de mortalidad asociada a la inactividad física en Bogotá. Revista Salud Pública, 8, 28-41.

Marques, S. (1995). Beneficios Psicológicos dela actividad física. Revista de psicología general yaplicada: Revista de la Federación Española de Asociaciones de Psicología, 48.

Martinson, B. C., O’Connor, P. J., \& Pronk, N. P. (2001). Physical Inactivity and Short-term All-Cause Mortality in Adults With Chronic Disease. Archives of Internal Medicine, 161, 1173-1180.

Martínez-González, M., Varo, J., Santos, J., De Irala, J., Gibney, M., Kearney, J., et al. (2001). Prevalence of physical activity during leisure time in the European Union. Medicine \& Science in Sports \& Exercise, 33, 1142-1146.

Instituto Estatal de las Mujeres (2005). Distribución de las defunciones por sexo de las cinco principales causas de muerte (edicion electrónica), [en línea]. Nuevo León: CEDAW.Disponible en: http:/ /www.nl.gob.mx/pics/pages/iem_cedaw_articulo12.base/ Cuadro23.pdf [2010, 2 de febrero].

Netz, Y. \& Raviv, S. (2004). Age Differences in Motivational Orientation Toward Physical Activity: An Application of Social-Cognitive Theory. Journal of Psychology, 138, 35-48.

National Health Service Information Centre, Lifestyle Statistics. (2009). Statistics on obesity, physical activity and diet: England, February 2009.

Pan American Health Organization. (2004). Calculation for agestandardized mortality for selected countries in Latin America and the Caribbean. Retrieved 30 de septiembre, 2008, from www.paho.org/English/AD/DPC/NC/svn-asmr-tables.htm
Pelletier, L., Dion, S., Tuson, K., \& Green-Demers, I. (1999). Why Do People Fail to Adopt Environmental Protective Behaviors? Toward a Taxonomy of Environmental Amotivation. Journal of Applied Social Psychology, 29, 2481-2504.

Porras-Sanchez, M. (2009). Hábitos y actitudes de los sevillanos ante el deporte 2008. Sevilla: Diseño Sur.

Ruiz-Juan, F., dela Cruz, E., \& Piéron, M. (2009). Actividad e inactividad física en adultos durante el tiempo libre. In F. Ruiz-Juan, GarcíaMontes, M., Piéron, M. (Ed.), Actividad física y estilos de vida saludables. Análisis de los determinantes de la práctica en adultos. (Primera ed., pp. 53-60). Almería, España: Wanceulen.

Ruiz-Juan, F., García-Montes, E., \& Piéron, M. (2009). Actividad física y estilos de vida saludables. Análisis de los determinantes de la práctica en adultos. Sevilla: Wanceulen.

Ruiz-Juan, F. \& García-Montes, M. (2005). Hábitos físico-deportivos de los almerienses en su tiempo libre. (Universidad de Almería. Servicio de publicaciones. ed. Vol. 43). Almería: Monografias. Humanidades.

Ruiz-Juan, F., García-Montes, E. \& Díaz, A. (2007). Análisis de las motivaciones de práctica de actividad física y de abandono deportivo en la Ciudad de La Habana (Cuba). Anales de psicología, 3, 152166.

Sallis, J., \& Owen, N. (1999). Physical Activity \& Behavioral Medicine (3 ed.). California: SAGE.

Seclén-Palacín, J. \& Jacoby, E. (2003). Factores sociodemográficos y ambientales asociados con la actividad física deportiva en la población urbana del Perú. Revista Panamericana de Salud Pública, 14, 255264.

Skinner, E. A., Wellborn, J. G., \& Connell, J. P. (1990). What It Takes to Do Well in School and Whether I've Got It: A Process Model of Perceived Control and Children's Engagement and Achievement in School. Journal of Educational Psychology, 82, 22-32.

Thøgersen-Ntoumani, C., Ntoumanis, N. \& Nikitaras, N. (2008). Typologies of Greek inactive older adults based on reasons for abstaining from exercise and conditions for change. Journal of Sports Sciences, 26, 1341-1350.

United States Department of Health and Human Services. (1996). Physical Activity and Health: A Report of the Surgeon General.

Varo, J. J., Martinez-Gonzalez, M. A., de Irala-Estevez, J., Kearney, J., Gibney, M., \& Martinez, J.A. (2003). Distribution and determinants of sedentary lifestyles in the European Union. International Journal of Epidemiology, 32, 138-146.

Vlachopoulos, S. P., \& Gigoudi, M.A. (2008). Why Don’t You Exercise? Development of the Amotivation Toward Exercise Scale Among Older Inactive Individuals. Journal of Aging \& Physical Activity, 16, 316-341.

Vuori, I., Oja, P., Cavill, N., \& Coumans, B. (2001). La Actividad Física para la mejora de la Salud. Guía Europea.

World Health Organization. (2003a). Canadian Community Health Survey: Statistics Canada.

World Health Organization. (2003b). Encuesta Nacional de Salud, Chile 2003. Santiago de Chile.

World Health Organization. (2008). Benefits of physical activity. Retrieved 30 de septiembre, 2008, de: www.who.int/ dietphysicalactivity/factsheet_benefits/en/print.html.

Wilcox, S., Castro, C., King, A., Housemann, R. \& Brownson, R. C. (2000). Determinants of leisure time physical activity in rural compared with urban older and ethnically diverse women in the United States. Journal of Epidemiology and Community Health, 54, 667-672.

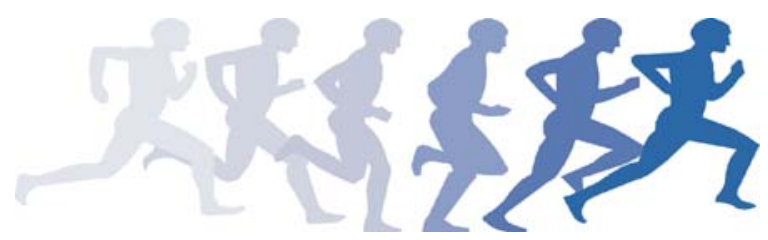

